\title{
In vitro evaluation of aortic valve prosthesis in a novel valved conduit with pseudosinuses of Valsalva
}

\author{
Ruggero De Paulis, MD, ${ }^{a}$ Christoph Schmitz, Dr-Ing, ${ }^{\text {b }}$ Raffaele Scaffa, MD, ${ }^{\text {a }}$ Paolo Nardi, MD, ${ }^{\text {a }}$ Luigi Chiariello, MD, \\ and Helmut Reul, Dr-Ing
}

Objective: This study was undertaken to determine whether the presence of vortices immediately above a prosthetic aortic valve could negatively influence the in vitro hydrodynamic performances of a biologic or mechanical valve implanted in a new Dacron polyester fabric conduit that incorporates sculpted sinuses of Valsalva.

Methods: With a computer-controlled pulse duplicator, the in vitro performance (pressure differences, closure and leakage volumes, and energy losses) of a 25-mm mechanical or biologic prosthesis implanted in a standard Dacron straight conduit or in the new Dacron graft with a sculpted sinus were analyzed and compared.

Results: The mechanical and biologic prostheses at $7 \mathrm{~L} / \mathrm{min}$ cardiac output showed pressure drops across the valve of $8.72 \mathrm{~mm} \mathrm{Hg}$ and $13.45 \mathrm{~mm} \mathrm{Hg}$, respectively, when inserted in the new Valsalva-style graft and of $7.97 \mathrm{~mm} \mathrm{Hg}$ and $12.94 \mathrm{~mm} \mathrm{Hg}$, respectively, when inserted in the standard graft. The closure and leakage volumes for mechanical valves were higher than those for biologic valves; however, the presence or absence of sinuses did not result in significant differences in closure and leakage volumes. The maximal total energy losses were $5.89 \%$ and $9.49 \%$ for mechanical and biologic valves, respectively. No differences were evident between the two different Dacron grafts for each prosthetic heart valve.

Conclusion: The normal opening and closing behavior of a prosthetic aortic valve was not altered or modified by a different root shape above the heart valve. The presence of vortices inside the pseudosinuses of Valsalva did not influence the hydrodynamic properties of the biologic and mechanical valves tested.

From the Cardiac Surgery Department, University of Tor Vergata, Rome, Italy, ${ }^{\mathrm{a}}$ and Helmholtz-Institute for Biomedical Engineering, RWTH Aachen, Aachen, Germany. ${ }^{\mathrm{b}}$

Received for publication Dec 31, 2004; revisions received April 14, 2005; accepted for publication April 22, 2005.

Address for reprints: Ruggero De Paulis, MD, Cattedra di Cardiochirurgia, Università di Roma Tor Vergata, European Hospital, via Portuense 700, 00149 Roma, Italy (E-mail: depauli@tin.it).

J Thorac Cardiovasc Surg 2005;130:1016-21 $0022-5223 / \$ 30.00$

Copyright (C) 2005 by The American Association for Thoracic Surgery

doi:10.1016/j.jtcvs.2005.04.028
$\mathrm{T}$ The evaluation of the hydrodynamic properties of prosthetic valves is a useful indicator of expected clinical performance. Numerous devices and mock circulations have been described for the measurement of in vitro performances of artificial heart valves. ${ }^{1-3}$ The computer-controlled pulse duplicator is a hydraulic model of the left human circulatory system and a well-established means of obtaining information on the in vitro performance of prosthetic heart valves. ${ }^{1,4}$ However, the opening and closing characteristic of a biologic or mechanical valve could potentially be modified by the presence or absence of vortices inside the aortic root immediately above the implanted valve.

We have recently introduced into clinical practice a new Dacron polyester fabric conduit (Gelweave Valsalva; Vascutek Terumo, Renfrewshire, United Kingdom) that incorporates sculpted sinuses of Valsalva and has been found particularly advantageous in both types of aortic valve-sparing operation, where it contributes to a more anatomically and physiologically accurate reconstruction of the aortic root. ${ }^{5,6}$ It has also been used in association with mechanical or biologic valves in a composite aortic root replacement type of aortic root reconstruction because of its improved adaptability to the patient's anatomy. ${ }^{7,8}$ In fact, its peculiar shape is characterized by a lower section that stretches horizontally and tends to reproduce 
the bulged section of the aortic root corresponding to the sinuses. In this way it reduces the tension on the coronary anastomoses once the aorta is under pressure and the heart resumes beating. Unlike in the aortic valve-sparing operation, in which the use of the new Dacron graft allows the reconstruction of three independent sinuses of Valsalva, when used as a composite aortic root replacement its final shape is that of a single, large, egg-shaped sinus to which the two coronary ostia are attached.

The aim of this study was to determine whether the presence of vortices inside this single sinus immediately above the prosthetic aortic valve could negatively influence the hydrodynamic in vitro performances of a prosthetic heart valve. We therefore analyzed and compared the in vitro performances of mechanical and biologic aortic valve prostheses inside a conventional straight Dacron conduit and inside the new Dacron graft that incorporates the sinuses of Valsalva.

\section{Materials and Methods}

The design of the pulse duplicator, shown in Figure 1, is based on the theoretic and experimental studies of Reul and colleagues. ${ }^{4}$ This device has been used in testing of several aortic prosthetic valves. ${ }^{9}$ To obtain physical similarity to the in situ flow process, the physiologic input and output impedances of the left human circulatory system are simulated by means of tuned hydraulic elements, such as adjustable compliances (Figure 1,13 and 15) and resistances (Figure 1,14 and 16). The flexible silicone ventricular model (Figure 1,9) and the aortic valved conduit (Figure 1, 10) are based on physiologic geometric data. ${ }^{10}$ The volume displacement of the simulated left ventricle is generated by an electrohydraulic drive unit (Figure 1, 17). The piston expands and contracts the flexible model ventricle by means of the fluid inside the compression chamber (Figure 1,7) according to a programmed volume-time function. An additional air volume serves as a variable ventricular compliance. The computer-generated and -controlled volume displacement signal facilitates a reliable and reproducible simulation of the natural ventricular dynamics. By means of frequency and piston amplitude variations, different circulatory conditions are adjustable.

Two types of aortic valve prostheses of the same diameter of 25 $\mathrm{mm}$, the St Jude mechanical prosthesis (St Jude Medical Inc, Minneapolis, Minn) and the Hancock II bioprosthesis (Medtronic, Inc, Minneapolis, Minn), were implanted in a standard 28-mm Dacron conduit (Vascutek Terumo, Renfrewshire, United Kingdom) or in a new 28-mm Valsalva-style Dacron graft and then inserted into the aortic position of the model. The design and characteristics of the modified Dacron graft have been described in detail elsewhere. ${ }^{7}$ In brief, it is composed of three portions: a standard Dacron graft, a short portion of the same Dacron graft with corrugation set perpendicularly (longitudinally) to the rest of the graft (called the skirt, with a length equal to the diameter of the graft), and a collar consisting of another short tract of a standard Dacron tube of the same diameter attached at the end of the skirt. The collar is needed to attach a valve prosthesis when performing a composite aortic root replacement. Under the pressure of blood, the tissue in the skirted portion of the graft will stretch horizontally to mimic the bulged portion of the natural aortic root.

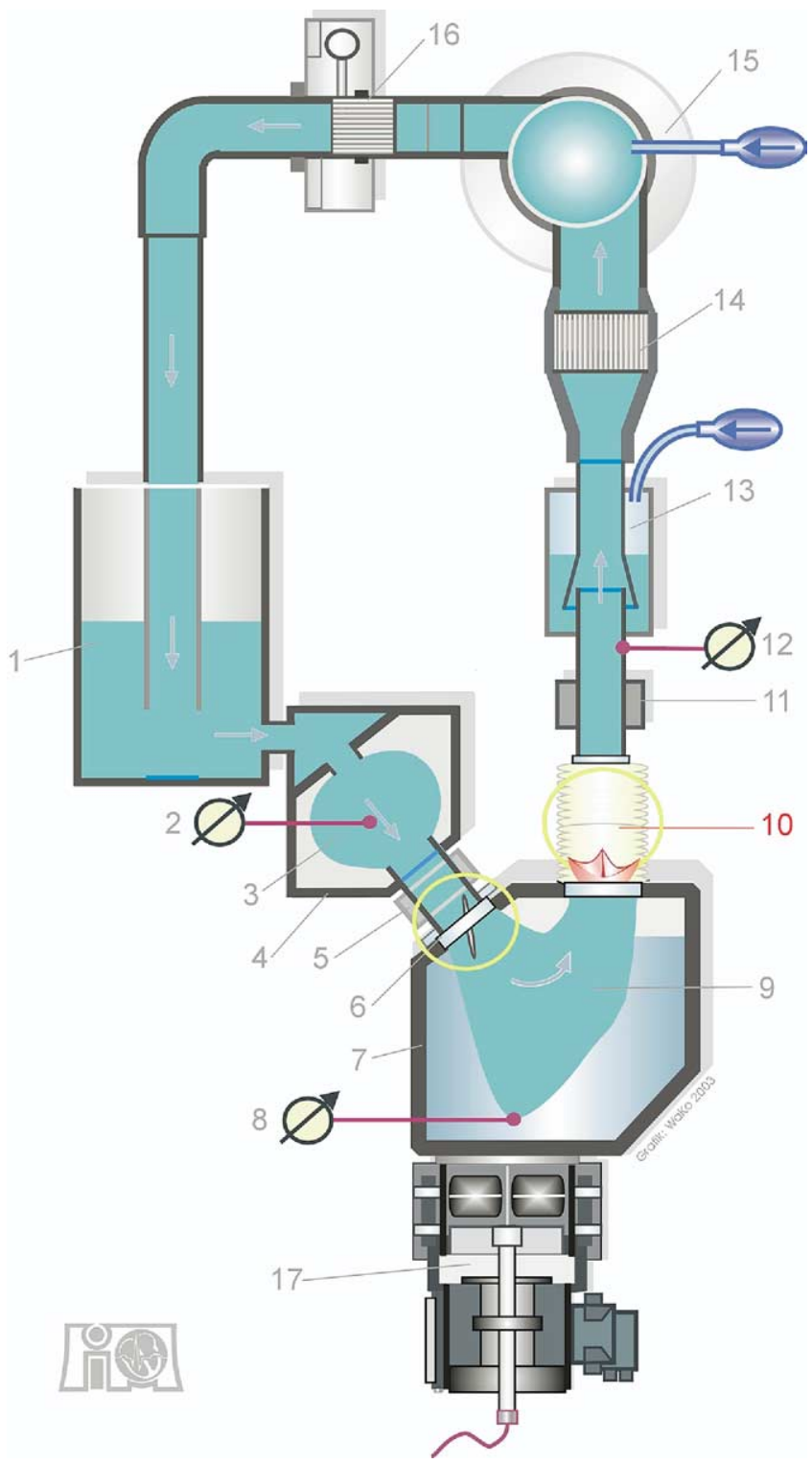

Figure 1. Schematic drawing of pulse duplicator used in experiment: reservoir (1), pressure transducers $(2,8,12)$, atrium (3), atrium chamber (4), flowmeters $(5,11)$, mitral valve (6), compression chamber with fluid and air compliance (7), silicone ventricle (9), aortic valve conduit (10), adjustable compliances $(13,15)$, resistance (14), adjustable resistance (16), electrohydraulic drive unit (17).

For determination of pressure, volume, and energy losses, pressure and flow signals are measured at several positions within the pulse duplicator. Pressures are recorded within the atrium (Figure 1,2), in the apex region of the ventricle (Figure 1,8), and in the ascending aorta (Figure 1, 12) with strain gauge pressure transducers (Cobe Laboratories, Inc, Lakewood, Colo). Flow is recorded by electromagnetic flowmeters (Zedepa Instruments, Seattle, Wash). The mitral flowmeter (Figure 1,5) is located up- 

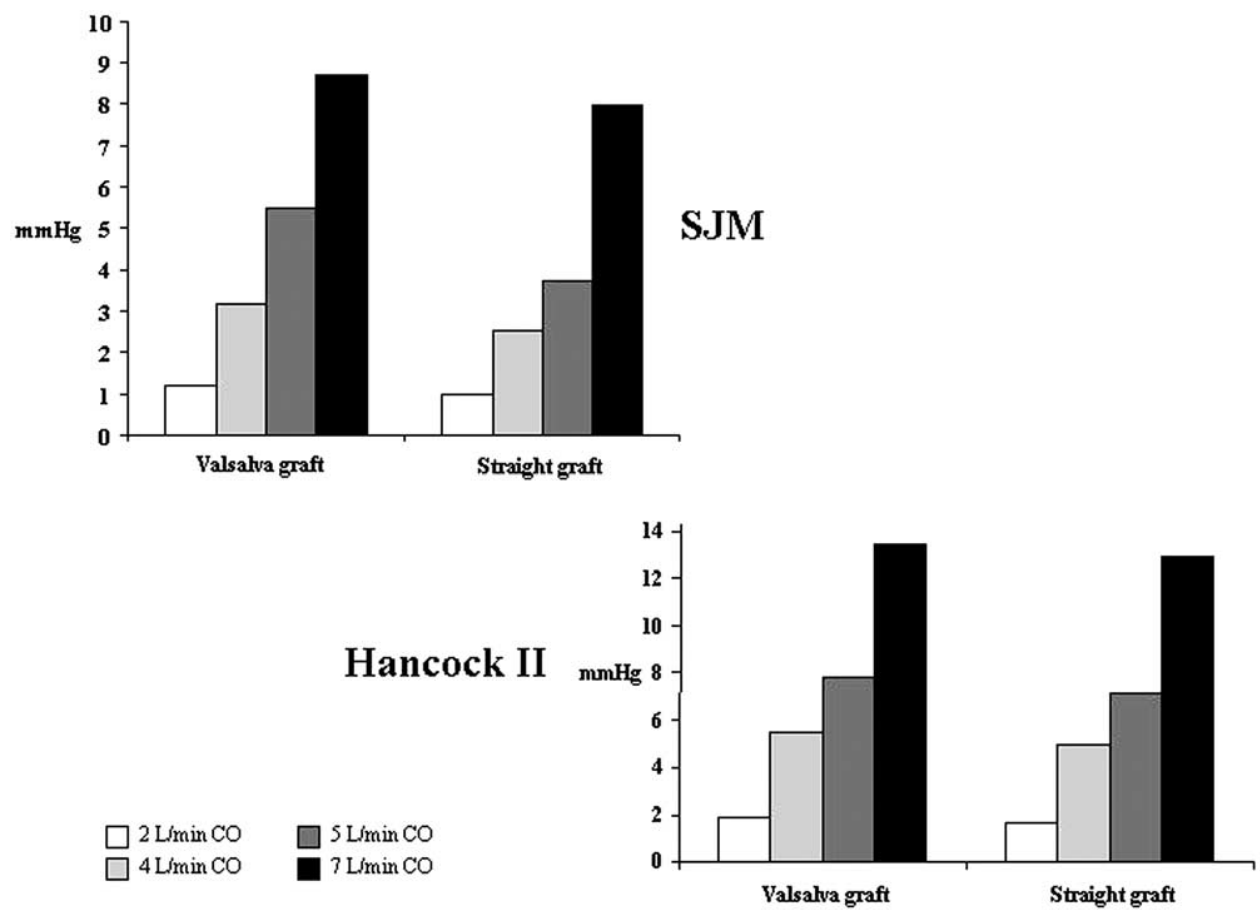

Figure 2. Mean systolic pressure differences at $\mathrm{COs}$ of 2, 4, 5, and $7 \mathrm{~L} / \mathrm{min}$ (averaged over 64 consecutive beats per Food and Drug Administration conditions) for 25-mm St Jude Medical (SJM) and 25-mm Hancock II valve prostheses.

stream of the mitral valve (Figure 1, 6). For the aortic valve (Figure 1,10), the flowmeter (Figure 1,11) is located 4 diameters downstream. The sensor signals are processed by amplifiers and special data-acquisition software. Each signal was averaged over 64 consecutive beats, and the averaged signals were then transferred to a second computer for off-line analysis. Therefore all reported values are mean values for 64 cycles. Mean systolic pressure difference, regurgitation volume, and energy losses were determined according to the Food and Drug Administration Replacement Heart Valve Guidance. ${ }^{11}$

\section{Mean Systolic Pressure Difference}

The mean systolic pressure difference $\Delta \bar{P}_{\mathrm{SYS}}$ is the time integral of ventricular pressure $\left(\mathrm{P}_{\mathrm{V}}\right)$ minus aortic pressure $\left(\mathrm{P}_{\mathrm{Ao}}\right)$ during the systolic period divided by systolic duration time $\left(\mathrm{T}_{\mathrm{SYS}}\right)$ :

$$
\Delta \overline{\mathrm{P}}_{\mathrm{SYS}}=\frac{1}{\mathrm{~T}_{\mathrm{SYS}}} \int_{\mathrm{T}_{\mathrm{SYS}}}\left(\mathrm{P}_{\mathrm{V}}-\mathrm{P}_{\mathrm{Ao}}\right) \mathrm{dt}
$$

\section{Closure and Leakage Volume}

The closure volume $\left(\mathrm{V}_{\mathrm{CL}}\right)$ and leakage volume $\left(\mathrm{V}_{\mathrm{L}}\right)$ equations represent the time integrals of aortic flow $(\dot{V})$ during closure and leakage periods, respectively.

$$
\mathrm{V}_{\mathrm{CL}}=\int_{\mathrm{T}_{\mathrm{CL}}} \dot{\mathrm{V}} \mathrm{dt}
$$

$$
\mathrm{V}_{\mathrm{L}}=\int_{\mathrm{T}_{\mathrm{L}}} \dot{\mathrm{V}} \mathrm{dt}
$$

The closure volume is defined as that part of the regurgitant volume that flows back through the valve during the time interval of valve closing $\left(\mathrm{T}_{\mathrm{CL}}\right)$, in the time needed to move the occluder from the open to the closed position. The leakage volume is defined as that part of the regurgitant volume that passes during the rest of the cycle $\left(\mathrm{T}_{\mathrm{L}}\right)$ and the closed state of the valve through the gap between the occluder and the valve ring.

\section{Energy Losses}

Energy loss $(\Delta \mathrm{E})$ is derived as the time integral of the product of mean pressure difference and aortic flow during defined time intervals, such as the systolic period (systolic energy loss), closure interval (closure energy loss), and leakage interval (leakage energy loss):

$$
\Delta \mathrm{E}=\int_{\mathrm{T}} \Delta \mathrm{P} \dot{\mathrm{V}} \mathrm{dt}
$$

All parameters were determined for four different cardiac outputs (COs): 2.0, 4.0, 5.0, and $7 \mathrm{~L} / \mathrm{min}$ at a fixed beat rate of 70 beats/min, resulting in stroke volumes of $28,57,71$, and $100 \mathrm{~mL}$ respectively. The systolic duration was $35 \%$ of the cycle period. The mean atrial and aortic pressures were kept constant at $10 \mathrm{~mm}$ $\mathrm{Hg}$ and $100 \mathrm{~mm} \mathrm{Hg}$, respectively, for all COs. 

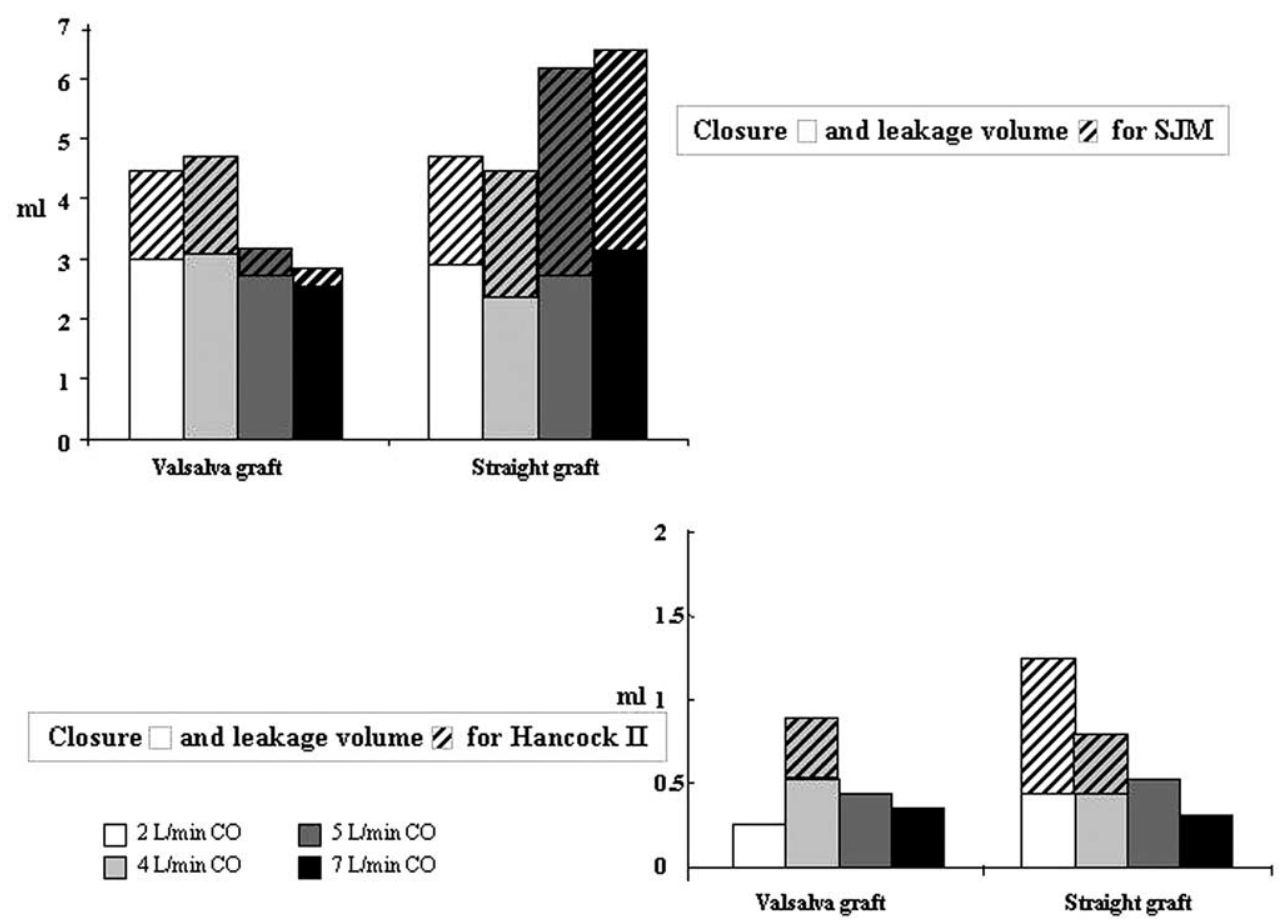

Figure 3. Closure and leakage volumes at $\mathrm{COs}$ of 2, 4, 5, and $7 \mathrm{~L} / \mathrm{min}$ (averaged over 64 consecutive beats per Food and Drug Administration conditions) for 25-mm St Jude Medical (SJM) and 25-mm Hancock II valve prostheses.

\section{Results}

\section{Pressure Differences}

The mean systolic pressure differences across the prosthetic heart valves at each $\mathrm{CO}$ are shown in Figure 2. The pressure drop across the valves increased with increasing flow rate. The mechanical valves generally had lower pressure differences (related to the smaller effective orifice opening area of the porcine valve). The bileaflet St Jude Medical valve showed similar pressure differences at the various COs when inserted in a Valsalva-type graft and in a standard straight graft, with peaks at a $\mathrm{CO}$ of $7 \mathrm{~L} / \mathrm{min}$ of $8.72 \mathrm{~mm} \mathrm{Hg}$ and $7.97 \mathrm{~mm} \mathrm{Hg}$, respectively. Similarly, the Hancock II bioprosthesis did not show any significant differences when inserted in a Valsalva-type graft or in a standard straight graft, with a peak values at a $\mathrm{CO}$ of $7 \mathrm{~L} / \mathrm{min}$ of $13.45 \mathrm{~mm}$ $\mathrm{Hg}$ and $12.94 \mathrm{~mm} \mathrm{Hg}$, respectively.

\section{Regurgitation (Closure and Leakage Volumes)}

Figure 3 shows both closure and leakage volumes that have been normalized to the respective stroke volumes at the aforementioned COs and a constant heart rate of 70 beats/ min. In Table 1, these values are expressed as percentage of stroke volume.

As expected, the closure and leakage volumes for mechanical valves were higher than those for biologic valves. The presence or absence of sinuses did not result in signif- icant differences in the closure and leakage volumes in either the mechanical or the biologic prosthetic heart valve. The lowest closure volume of the 25-mm St Jude Medical prosthetic valve was $2.4 \mathrm{~mL} / \mathrm{beat}$ in the standard straight graft at a $\mathrm{CO}$ of $4 \mathrm{~L} / \mathrm{min}$, whereas the lowest closure volume for the Hancock valve was $0.2 \mathrm{~mL} / \mathrm{beat}$ in the Valsalva-type

TABLE 1. Closure and leakage volumes expressed as percentage of stroke volume for the 25-mm St Jude Medical and 25-mm Hancock II valve prostheses

\begin{tabular}{lccccc}
\hline & \multicolumn{2}{c}{$\begin{array}{c}\text { Graft with sinuses } \\
\text { (\% SV) }\end{array}$} & & \multicolumn{2}{c}{$\begin{array}{c}\text { Straight graft } \\
\text { (\% SV) }\end{array}$} \\
\cline { 2 - 3 } \cline { 5 - 6 } CO (L/min) & Closure & Leakage & & Closure & Leakage \\
\hline St Jude Medical & & & & & \\
2 & 10.35 & 5.07 & & 10.25 & 2.86 \\
4 & 5.46 & 2.82 & & 4.2 & 4.13 \\
5 & 3.95 & 0.45 & & 2.88 & 4.69 \\
7 & 2.54 & 0.28 & & 3.15 & 3.21 \\
Hancock II & & & & & \\
2 & 0.7 & 0 & & 1.28 & 2.79 \\
4 & 0.88 & 0.64 & & 0.8 & 0.5 \\
5 & 0.61 & 0 & & 0.74 & 0 \\
7 & 0.33 & 0 & & 0.25 & 0 \\
\hline
\end{tabular}

$S V$, Stroke volume. 


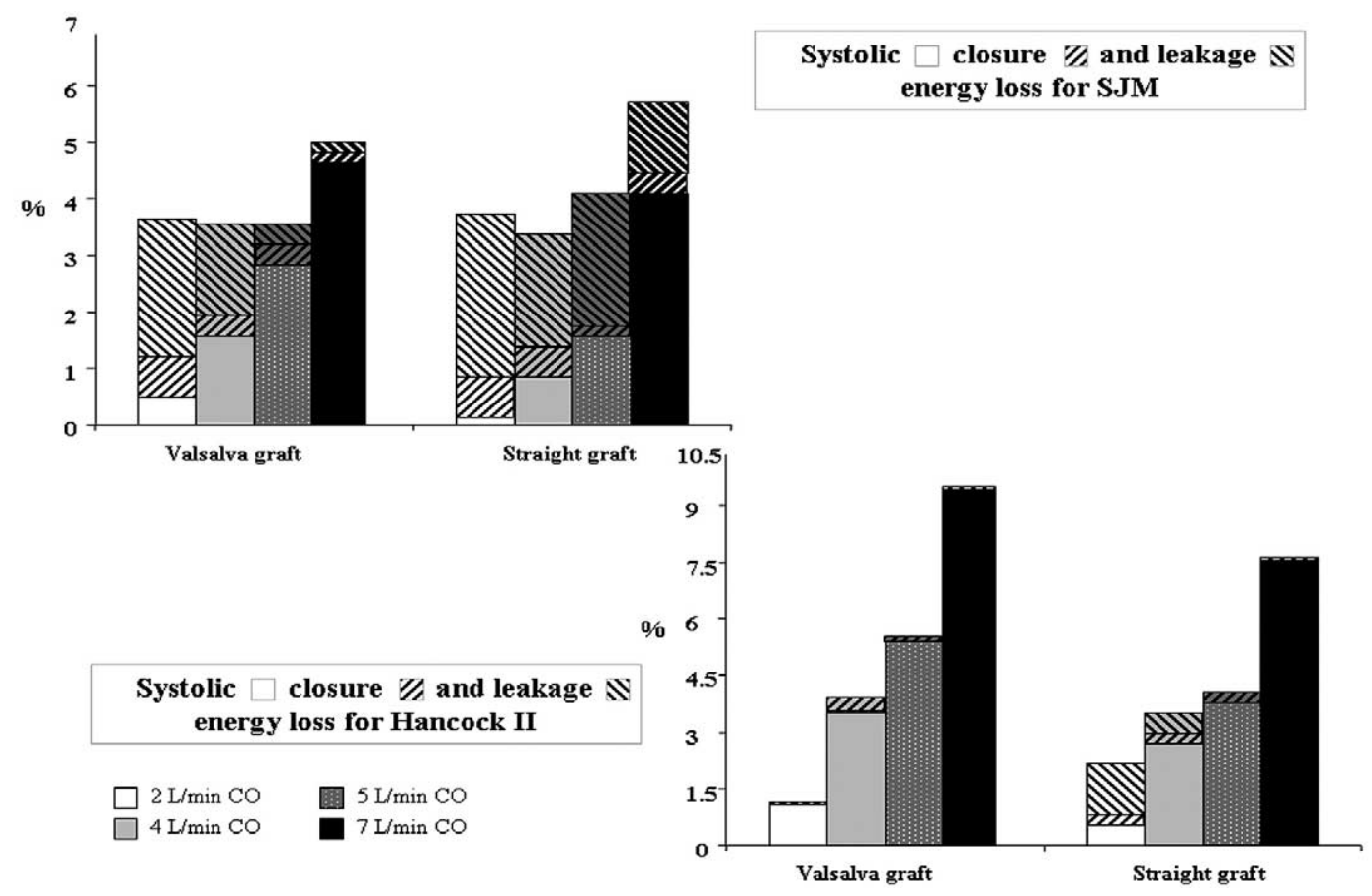

Figure 4. Accumulated energy losses (systolic, closure, and leakage) at COs of 2, 4, 5, and $7 \mathrm{~L} / \mathrm{min}$ (averaged over 64 consecutive beats per Food and Drug Administration conditions) for 25-mm St Jude Medical (SJM) and 25-mm Hancock II valve prostheses.

graft at a $\mathrm{CO}$ of $2 \mathrm{~L} / \mathrm{min}$. Similarly, the lowest leakage volume of the St Jude Medical valve was $0.32 \mathrm{~mL} / \mathrm{beat}$ at a $\mathrm{CO}$ of $5 \mathrm{~L} / \mathrm{min}$ in the Valsalva-type graft, whereas it was 0 $\mathrm{mL} /$ beat for the Hancock valve in both grafts at various COs.

\section{Energy Loss}

Figure 4 depicts the accumulated transvalvular energy losses for the systolic phase, the closing phase, and the leakage phase for the mechanical and biologic valves. The maximal total energy losses were $5.89 \%$ and $9.49 \%$ for mechanical and biologic valves, respectively. No differences were evident between the two different Dacron grafts for each prosthetic heart valve.

\section{Discussion}

Major problems associated with tissue and mechanical aortic valve replacements include thrombosis, hemolysis, tissue overgrowth, infection, calcification, valve wear, and excessive pressure gradients. Despite these potential complications, the prognosis for patients receiving an aortic prosthetic heart valve remains good, with excellent long-term survival and a good quality of life.

The simultaneous replacement of the aortic valve and the entire ascending aorta with coronary reimplantation (composite aortic root replacement) ${ }^{12}$ has become a standard operation for all cases of aortic valve disease associated with aneurysm or dissection of the aortic root and ascending aorta. Many modifications of the standard surgical technique have been described through the years. The popularity and the increased need for this type of operation have stimulated the development of composite valved conduits to facilitate and expedite the surgical procedure. The use of composite grafts has shown excellent long-term results with an extremely low rate of complications. ${ }^{13,14}$

In recent years, we introduced into clinical practice an originally designed Dacron conduit with the aim of obtaining a more anatomically accurate reconstruction of the aortic root. ${ }^{7}$ In particular, this newly designed conduit tends to reproduce the bulged section of the aortic root corresponding to the sinuses of Valsalva. The importance of the sinuses of Valsalva in regulating the aortic valve motion during the cardiac cycle has been extensively studied in recent years. ${ }^{15}$ By using this Valsalva-type Dacron conduit, it was possible to obtain more physiologically accurate valve motion characteristics after either the remodeling or the reimplantation type of valve-sparing procedure. ${ }^{5,6} \mathrm{Be}-$ cause of its peculiar shape, this modified Dacron graft has also been used in composite aortic root replacement, where it might facilitate the surgical technique and at the same time decrease the tension on coronary ostia anastomoses. As 
a consequence, its practical advantages would be more evident in those cases where the coronary ostia are more difficult to mobilize (eg, reoperative cases, calcification, and infection). In the case of composite aortic root replacement, this new vascular prosthesis does not reproduce three independent sinuses but rather a single, large, egg-shaped proximal portion. Although vortices are present within this single egg-shaped sinus, their effects on the mobile elements of mechanical or biologic valve prosthesis are less evident and more difficult to visualize.

For these reasons, we attempted to determine whether the presence of vortices generated inside this initial portion of the Dacron graft could somehow affect the hydrodynamic performance of a mechanical or a biologic valve. In fact, the presence of nonlaminar flow immediately above the prosthetic heart valve could potentially influence the opening angle of a mechanical valve, with a direct effect on the transvalvular gradient. A change in the opening angle might also have a direct effect on the closure volume, because a shorter time for valve closure leads to smaller closure volumes.

However, the results of these experiments showed that the differences in flow pattern immediately above the prosthetic heart valve did not modify or alter the normal opening and closing behavior of the valve and thus hydrodynamic performance. In particular, the presence of pseudosinuses of Valsalva in the new conduit did not appear to influence the hydrodynamic properties of prosthetic mechanical valves (pressure drop and energy loss across the valves, as well as the amount of back flow during and after the valve is closed). Svensson ${ }^{16}$ recently noted that the gradient across composite valves appears to be greater than for equivalent "noncomposite" aortic valve sizes, suggesting the possibility that the presence of sinuses or the maintenance of a certain root elasticity could have a positive effect on postoperative gradients.

Similarly to the mechanical valves, the presence of vortices inside the newly reconstructed root did not appear to have any influence on the hydrodynamic behavior of biologic valve prostheses. In fact, the total energy loss, which can be considered a measure of the efficiency of the valve, was not different between a standard conduit and one with pseudosinuses of Valsalva. In particular, in both Dacron grafts the total energy loss for the biologic valve was due almost exclusively to the systolic energy losses, whereas closure and leakage energy losses were extremely small or absent.
In conclusion, this series of experiments clearly showed that the presence of vortices inside a single, large, eggshaped portion of ascending aortic graft immediately above the aortic valve prosthesis did not modify the hydrodynamic performances of the biologic and mechanical valves tested. The advantages of the improved anatomic adaptability that is peculiar to this Valsalva-type graft can therefore be exploited without the risk of affecting prosthetic valve performance.

\section{References}

1. Reul H. Cardiovascular simulation models. Life Support Syst. 1984;2: 77-98.

2. Farahifar D, Cassot F, Bodard H, Pellissier R. Velocity profiles in the wake of two prosthetic heart valves using a new cardiovascular simulator. J Biochem. 1985;18:789-802.

3. Fisher J, Jack GR, Wheatley DJ. Design of a function test apparatus for prosthetic heart valves: initial results in the mitral position. Clin Phys Physiol Meas. 1986;7:63-73.

4. Reul H, Minamitami H, Runge J. A hydraulic analog of the systemic and pulmonary circulation for testing artificial hearts. Proc ESAO. 1975;2:120-7.

5. De Paulis R, De Matteis GM, Nardi P, Scaffa R, Buratta MM, Chiariello L. Opening and closing characteristics of the aortic valve after valve-sparing procedures using a new aortic root conduit. Ann Thorac Surg. 2001;72:487-94.

6. De Paulis R, De Matteis GM, Nardi P, Scaffa R, Bassano C, Chiariello L. Analysis of valve motion after the reimplantation type of valvesparing procedure (David I) with a new aortic root conduit. Ann Thorac Surg. 2002;74:53-7.

7. De Paulis R, De Matteis GM, Nardi P, Scaffa R, Colella D, Chiariello L. A new aortic Dacron conduit for surgical treatment of the aortic root pathology. Ital Heart J. 2000;1:457-63.

8. De Paulis R, Bassano C, Scaffa R, Nardi P, Bertoldo F, Chiariello L. Bentall procedures with a novel valved conduit incorporating sinuses of Valsalva. Surg Technol Int. 2004;12:195-201.

9. Knott E, Reul H, Knoch M, Steinseifer U, Rau G. In vitro comparison of aortic heart valve prostheses. J Thorac Cardiovasc Surg. 1988;96: 952-61.

10. Swanson WM, Clark RE. Dimensions and geometric relationships of the human aortic valve as a function of pressure. Circ Res. 1974;35: 871-2.

11. Food and Drug Administration. Replacement heart valves: guidelines for data to be submitted to the Food and Drug Administration in support of applications premarket approval. Bethesda (Md): The Administration; 1982.

12. Bentall HH, de Bono A. A technique for complete replacement of the ascending aorta. Thorax. 1968;23:338-9.

13. Sioris T, David TE, Ivanov J, Armstrong S, Feindel CM. Clinical outcomes after separate and composite replacement of the aortic valve and ascending aorta. J Thorac Cardiovasc Surg. 2004;128:260-5.

14. Gott VL, Cameron DE, Alejo DE, Greene PS, Shake JG, Caparrelli DJ, et al. Aortic root replacement in 271 Marfan patients: a 24-year experience. Ann Thorac Surg. 2002;73:438-43.

15. Grande-Allen KJ, Cochran RP, Reinhall PG, Kunzelmann KS. Recreation of sinuses is important for sparing the aortic valve: a finite element study. J Thorac Cardiovasc Surg. 2000;119:753-63.

16. Svensson L. Invited commentary to: Complete aortic root replacement in patients with small aortic annulus. Ann Thorac Surg. 2002;73:728-9. 